\title{
Thakker-Donnai syndrome
}

INSERM

\section{Source}

INSERM. (1999). Orphanet: an online rare disease and orphan drug data base. Thakker-

Donnai syndrome. ORPHA:1780

Thakker-Donnai syndrome is a rare, genetic, lethal, multiple congenital

anomalies/dysmorphic syndrome characterized by facial dysmorphism (including long,

downward slanting palpebral fissures, hypertelorism, posteriorly rotated ears, broad

nasal bridge, short nose with a bulbous tip and anteverted nares, downturned corners of the mouth) as well as vertebral (occult spina bifida, hemivertebrae), brain (ventricular

dilatation, agenesis of corpus callosum), cardiac (teralogy of Fallot, ventricular septal

defect) and gastrointestinal (short esophagus with intrathoracic stomach, small intestine, spleen and pancreas, anal atresia) malformations. There have been no further descriptions in the literature since 1991. 